\title{
The Anesthesiologist, Rather Than the Anesthesia, May Influence the Outcomes following Stroke Thrombectomy
}

1 is more than likely that systemic hypotension resulting from anesthesia, regardless of the technique used, can have deleterious effects in patients with ongoing acute ischemic stroke. Cerebral autoregulation is often impaired in these neurocritical patients, rendering cerebral blood flow entirely dependent on cerebral perfusion pressure. Hence, maintaining an optimal mean arterial blood pressure may be vital to improving survival outcomes. On the other hand, cerebral vasoreactivity to $\mathrm{CO}_{2}$ is one of the most effective mechanisms to regulate the diameter of the cerebral arterioles. According to the Hagen-Poiseuille equation, the laminar flow rate is directly related to the differential pressure of the cerebral arterioles and the fourth power of the radius of these vessels. Consequently, optimizing arterial blood pressure while maintaining normocarbia should be one of the main goals when anesthetizing patients undergoing stroke thrombectomy to improve collateral circulation.

Not unexpectedly, Schönenberger et $\mathrm{al}^{1}$ have recently reported that in the Sedation versus Intubation for Endovascular Stroke Treatment (SIESTA) trial, which involved a cohort of 104 patients with acute ischemic stroke in the anterior circulation randomized to undergo either conscious sedation or general anesthesia, there was no significant difference in the neurologic improvement at 24 hours after the admission, measured by the National Institutes of Health Stroke Scale score. Furthermore, the same research group conducted a post hoc analysis of the data and reported in a recent issue of $A J N R$ that the collateral status, which was strongly related to the thrombectomy success in the SIESTA trial, was not significantly influenced by the anesthetic technique used. ${ }^{2}$

There is ongoing debate as to whether patients undergoing stroke thrombectomy would benefit from conscious sedation or general anesthesia. In the SIESTA trial, the systolic blood pressure and end-tidal carbon dioxide were closely monitored and maintained in the range of $120-180$ and $35-45 \mathrm{~mm} \mathrm{Hg}$, respectively. A

http://dx.doi.org/10.3174/ajnr.A5430 tight control of these variables, however, decreases the external validity of the results, as maintaining blood pressure and arterial partial pressure of carbon $(\mathrm{PaCO} 2)$ within physiologic ranges largely depends on the anesthesiologist's skills. Consequently, the potential advantages of general anesthesia may also be obscured by poor control of these crucial variables. Further clinical trials evaluating the variation of mean arterial blood pressure and $\mathrm{CO} 2$ between anesthetic techniques, are needed to elucidate the role of these critical aspects in the neurological and functional outcomes of patients undergoing stroke thrombectomy.

While general anesthesia can be associated with cardiovascular instability in neurocritical patients, anesthesiologists play a very important role in preventing a substantial drop in blood pressure following the induction in most cases, while maintaining normocarbia. Induction techniques have also evolved to provide highquality anesthesia in a quick and safe manner in the emergency environment, thus saving precious time for the neuroradiologist to perform a successful thrombectomy. On the other hand, patients undergoing thrombectomy under conscious sedation should be carefully selected because those with poor neurologic status are unlikely to cooperate and those having potentially difficult airways cannot always be safely sedated, especially considering that in this scenario, the airway access may be challenging.

\section{REFERENCES}

1. Schönenberger S, Uhlmann L, Hacke W, et al. Effect of conscious sedation vs general anesthesia on early neurological improvement among patients with ischemic stroke undergoing endovascular thrombectomy: a randomized clinical trial. JAMA 2016;316:1986-96 CrossRef Medline

2. Schönenberger S, Pfaff J, Uhlmann L, et al. The impact of conscious sedation versus general anesthesia for stroke thrombectomy on the predictive value of collateral status: a post hoc analysis of the SIESTA trial. AJNR Am J Neuroradiol 2017;38:1580-85 CrossRef Medline

(1)W. Fandino

The Walton Centre National Health Service Foundation Trust Liverpool, United Kingdom 